\title{
DiSEÑO E IMPLEMENTACIÓN DE UN PROTOTIPO DE TARJETA DIGITALIZADORA PARA MONITOREO TERMOMÉTRICO EN FUENTES TERMALES Y CAMPOS FUMARÓLICOS EN ÁREAS DE INFLUENCIA VOLCÁNICA
}

\author{
DESIGN AND IMPLEMENTATION OF A PROTOTYPE \\ OF DIGITALIZING CARD FOR THERMOMETRIC MONITORING \\ IN THERMAL SOURCES AND SMOKE FIELDS IN AREAS \\ OF VOLCANIC INFLUENCE
}

\begin{abstract}
Andrea Lorena Bravo Suarez ${ }^{1}$, Jorge Edison Mora Arroyo ${ }^{2}$
Servicio Geológico Colombiano-Observatorio Vulcanológico y Sismológico de Pasto. Pasto-Colombia.
\end{abstract}

Recibido: 30/04/2017 • Aprobado: 25/05/2017

\section{RESUMEN}

Para el Servicio Geológico Colombiano (SGC), a través de su Observatorio Vulcanológico y Sismológico de Pasto (OVSP), es una necesidad imperante la instrumentalización para el seguimiento térmico permanente, y en tiempo real, de fuentes termales y campos fumarólicos ubicados en zonas de amenaza volcánica, razón por la cual en este trabajo se presenta el diseño, e implementación, de un prototipo de tarjeta digitalizadora de datos de temperatura, para el acondicionamiento electrónico de señal de una termocupla tipo $K$. El prototipo permite la medición de temperaturas en el rango de $(-55 a+125)^{\circ} \mathrm{C}$ con una precisión de $10 \mathrm{mV} /{ }^{\circ} \mathrm{C}$, y se utiliza un data logger digital patronado que cuenta con su respectivo certificado de calibración para verificar el funcionamiento de la digitalización, y, por ende, de la lectura de temperatura. Se elabora el diseño de la tarjeta, previa ingeniería de requisitos, se valida su funcionamiento mediante simulación, y finalmente se implementa y se hacen las respectivas pruebas de laboratorio para posteriormente observar su comportamiento en campo, en la estación Mallama de la red de vigilancia del volcán Azufral. En general, los resultados obtenidos son satisfactorios, puesto que el error promedio absoluto obtenido en la medición de temperatura en condiciones controladas es de $1^{\circ} \mathrm{C} y$ en campo en ambientes de alta corrosión y acidez es de $2^{\circ} \mathrm{C}$. Lo anterior permite contribuir a la generación de un modelo que se pueda usar en las estaciones remotas termométricas que hacen parte de la red instrumental del SGC-OVSP.

Palabras clave: digitalización, error promedio absoluto, estación termométrica, prototipo, sensor, tarjeta, temperatura, termocupla.

\footnotetext{
${ }^{1}$ bravolorena94@outlook.com, https://orcid.org/0000-0002-6924-1414

2 jmoralsgc.gov.co, https://orcid.org/0000-0003-3850-4506
} 


\section{Abstract}

For the Geological Survey of Colombia (SGC), through its Volcanological and Seismological Observatory of grass (OVSP), is a prevailing need, instrumentation for permanent thermal monitoring in real time, thermal springs and Fumarólicos fields located in areas of volcanic threat, reason why this paper presents the design, and implementation of a prototype of a temperature data digitizer card for the electronic conditioning of a thermocouple signal type $K$. The prototype allows the measurement of temperatures in the range of $(-55 \text { to } 125)^{\circ} \mathrm{C}$ with precision of $10 \mathrm{mV} /{ }^{\circ} \mathrm{C}$, and a digital patterned data logger is used which has its respective calibration certificate to verify the operation of the digitization and, therefore, the temperature reading. The design of the card is elaborated after engineering of requirements, its operation is validated by simulation, and finally it is implemented and the respective laboratory tests are done to later observe its behavior in the field, in the Mallama station of the Azufral volcano surveillance network. In general, the results obtained are satisfactory, since the absolute average error obtained in the measurement of temperature under controlled conditions is $1{ }^{\circ} \mathrm{C}$ and in environments of high corrosion and acidity is $2^{\circ} \mathrm{C}$. This allows to contribute to the generation of a model that can be used in the remote Thermometric stations that are part of the instrument network of the SGC-OVSP.

Key words: absolute average error, card, digitalization, prototype, sensor, temperature, thermocouple, thermometric station.

\section{INTRODUCCIÓN}

La medición de la temperatura, como parte del proceso de investigación del fenómeno volcánico, constituye un insumo importante para la caracterización de parámetros geoquímicos propios de la dinámica de un volcán (López, 2007). En vista de que las erupciones volcánicas ocurren sin previo aviso, se convierten en fenómenos bastante interesantes de estudiar y muy complejos de predecir. En este contexto, la instrumentalización para el seguimiento termométrico permanente, implica, además de técnicas de procesamiento que soporten las condiciones ambientales y la corrosión debida al entorno, adquirir información en tiempo real de fuentes termales y campos fumarólicos, para aportar al cumplimiento de los objetivos misionales del Servicio Geológico Colombiano (SGC), a través de su Observatorio Vulcanológico y Sismológico de Pasto (OVSP).

Cabe destacar que la exactitud y precisión en la medición de temperatura, se ve afectada por las condiciones ambientales en que se efectúa la medida, así como los sensores, método y, transmisores utilizados en el proceso de medición y el ruido electrónico del digitalizador (Schmid, 2004).
En estudios similares, generalmente se emplean microcontroladores (entre ellos PIC), no solo en la etapa de control sino también para la conversión análogadigital, ya que permiten la programación, el control y la sincronización de tareas electrónicas. En Meléndez (2008) y Carrion (2015), por ejemplo, se utiliza un microcontrolador para el diseño de un sistema de medición de temperatura en un secador solar para madera y en una piscina camaronera. Por su parte en Quijada (2011), se diseña un data logger para medir temperatura haciendo uso del PIC 18F4550 con resultados satisfactorios. Finalmente, en López (2007) se desarrolla un sistema de monitoreo volcánico que incluye medición de temperatura por medio del integrado AD595 proporcionando una alternativa viable para una estación multiparamétrica.

Así, en este artículo se expone una metodología para diseñar un prototipo de tarjeta digitalizadora de temperatura, para monitoreo termométrico en estaciones termales y campos fumarólicos ubicados en zonas de influencia volcánica. En primer lugar, se definen los requerimientos que debe cumplir el prototipo, se 
diseña, simula e implementa, para posteriormente hacer las respectivas pruebas de laboratorio y en campo en la estación Mallama de la red de vigilancia del volcán Azufral de la red de monitoreo del SGC-OVSP.

Por lo tanto, el proyecto se considera relevante dentro de las áreas de electrónica y geoquímica de la institución, puesto que cuentan con tarjetas industriales para la adquisición de datos de temperatura, pero no poseen un diseño exclusivo y propio de las mismas. Además, el resultado puede extender su campo de aplicación a lugares en los que no se supere el rango de medición de temperatura del sensor y que no necesariamente sea de origen volcánico, como por ejemplo un invernadero o el control de hornos.

\section{Metodología}

\subsection{Diseńo del prototipo}

El grupo de investigación en instrumentación electrónica y telecomunicaciones del OVSP, establece los requerimientos que debe cumplir el prototipo: desplegar un menú de inicio con la presentación de la tarjeta, un menú de configuración que permita modificar la identificación (ID) de la estación (únicamente debe contener cuatro caracteres alfanuméricos), y la rata de muestreo de la señal (valor en segundos en un rango específico), adicionalmente debe contar con un reset físico.

En cuanto a hardware, se incluyen dos sensores para medir la temperatura, el primero es un LM35 que se utiliza para monitorear la temperatura ambiente, cuenta con salida lineal de $10 \mathrm{mV} /{ }^{\circ} \mathrm{C}$ en un rango de trabajo de $(-55 \mathrm{a}+150){ }^{\circ} \mathrm{C}$ y una resolución aproximada de $\pm 0.25^{\circ} \mathrm{C}$, además no requiere de circuitos adicionales para calibración externa, mientras que el segundo, que es una termocupla didáctica-genérica tipo $\mathrm{K}$, posee una sensibilidad de $40.8 \mu \mathrm{V} /{ }^{\circ} \mathrm{C}$ a $0^{\circ} \mathrm{C}$, permite monitorear la temperatura de un determinado punto térmico y requiere de un acondicionamiento de señal, que en este caso se realiza mediante el módulo AD595, que proporciona una salida analógica lineal de $10 \mathrm{mV} /{ }^{\circ} \mathrm{C}$ en un rango de trabajo de $(-55 \mathrm{a}+125)^{\circ} \mathrm{C}$ y cuya configuración y manipulación necesarias se realizaron con base en lo propuesto por Adeyeri (2012). El control de la tarjeta digitalizadora se hace mediante el PIC 18F4553 de Microchip que posee un conversor análogo-digital de 12 bits, es de bajo costo, desempeño eficiente, gran documentación y fácil adquisición en el mercado (Microchip, 2007).

En cuanto a software, el diagrama de flujo se muestra en la Figura 1, y su programación se realiza en el entorno de Mplab. El menú de inicio presenta la tarjeta con el título: "Tarjeta Digitalizadora Termocupla SGC-OVSP Firmware V.1”, y, a continuación, las dos opciones de iniciación: 1. Transmisión de datos y 2. Configuración de la tarjeta.

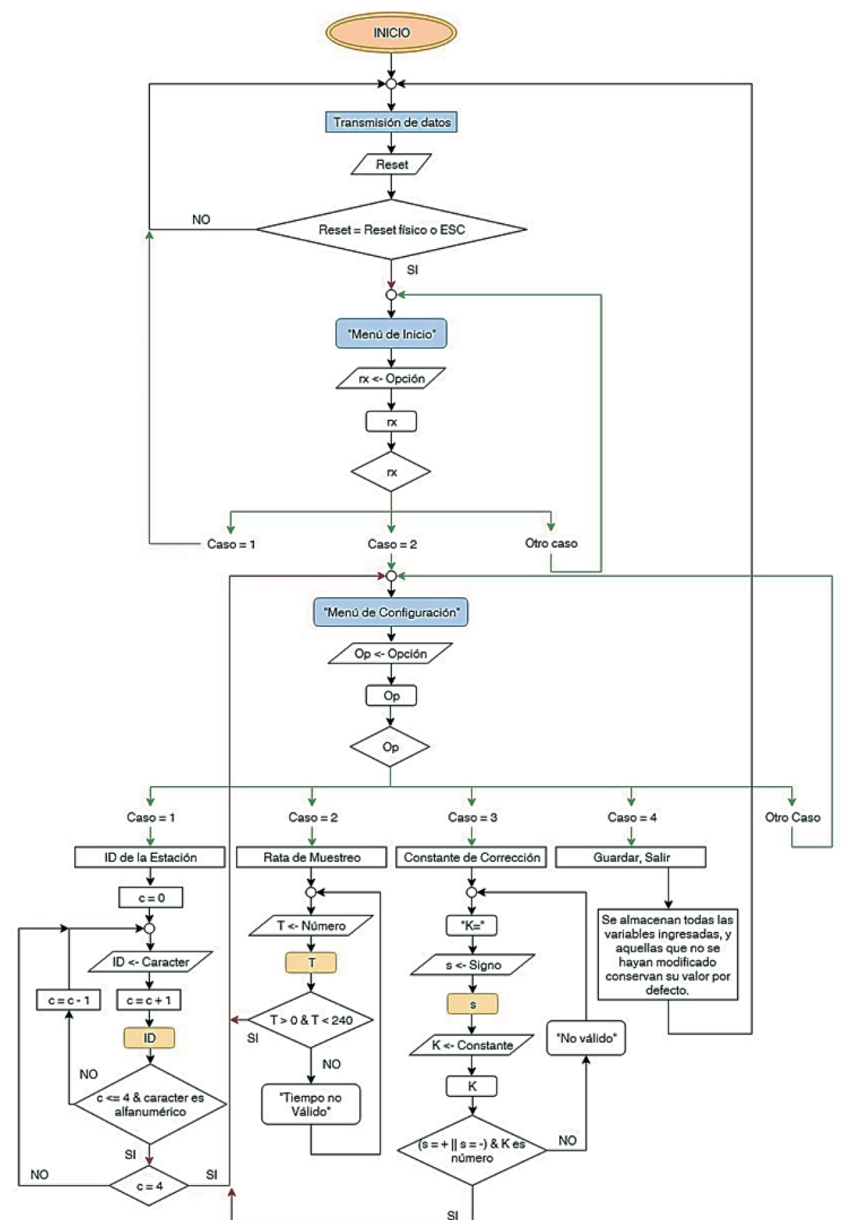

Fig. 1. Diagrama de flujo del código de programación que se compila en Mplab para el funcionamiento del prototipo de tarjeta digitalizadora de temperatura.

Fuente: autores 
Diseño e implementación de un prototipo de tarjeta digitalizadora para monitoreo termométrico en fuentes termales y campos fumarólicos en áreas de influencia volcánica

\subsubsection{Transmisión de datos}

Se leen los parámetros de configuración almacenados en la Eeprom del PIC y las variables físicas a medir, para posteriormente transmitir la cadena de datos por el puerto RS-232 de transmisión serial, como se indica en la Figura 2. Los parámetros asignados por defecto son, para la ID: BASE, la constante de corrección tiene un valor de 0 , y el tiempo de muestreo: 10 segundos.

El parámetro número 2 es de carácter binario, 1 indica la conexión normal del sensor mientras que el valor de 0 representa el circuito abierto en la termocupla. La señal del voltaje de

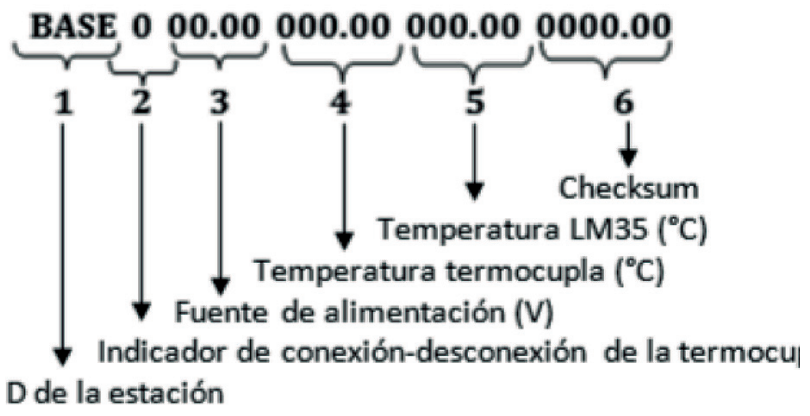

Fig. 2. Cadena de datos que se transmite con el prototipo de tarjeta digitalizadora.

Fuente: autores.

alimentación se toma a partir de un divisor de tensión de la fuente nominal de $12 \mathrm{~V}$, con una relación de $1 / 100$. Éste valor, junto con la temperatura se presentan con una precisión de dos cifras decimales y por último el parámetro de checksum hace referencia a la suma de verificación entre el valor de temperatura del LM35 y la termocupla, que se computa internamente.

Para el proceso de transmisión serial se configura el módulo EUSART del microcontrolador, utilizando la librería USART de Mplab. Se realiza una transmisión en modo asíncrono, en paquetes de 8 bits a 9600 baudios, con un error menor en la transmisión.

\subsubsection{Configuración}

Permite modificar la ID de la estación, la constante de corrección para el valor de temperatura de la termocupla $(\mathrm{K}=$ número entero $+\mathrm{o}-$ que se adiciona o se resta al valor digitalizado de la salida del AD595), y el tiempo de muestreo (números enteros uno a uno en el rango de (1 a 240 s), además de guardar cambios e inmediatamente iniciar la transmisión de datos.

Posteriormente, se desarrolla un esquema circuital en el software Proteus, que permite cargar el código compilado en el PIC y simular el funcionamiento del sistema en su totalidad, con el fin de corroborar el funcionamiento de la tarjeta.

\subsection{Implementación del prototipo}

Una vez realizada la simulación y depuración del software, se procede con las primeras pruebas del prototipo por medio de la implementación en protoboard. El esquema de conexiones utilizado se muestra en la Figura 3.

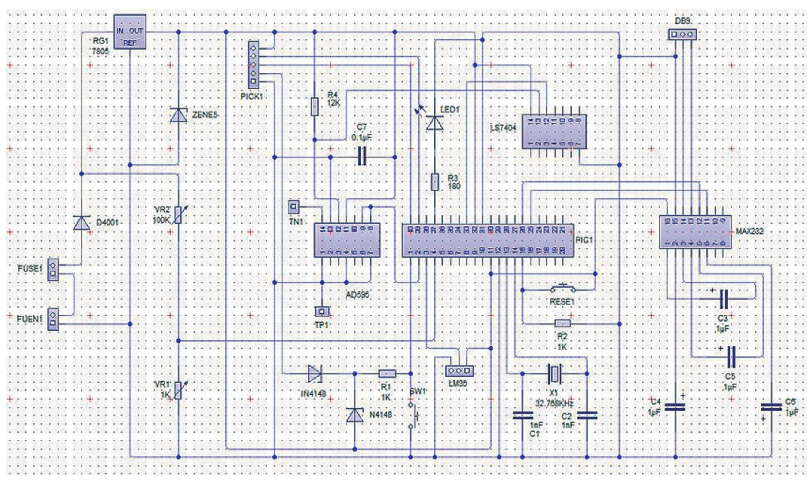

Fig. 3. Esquema de conexiones desarrollado en multisim para la implementación en protoboard y PCB del prototipo de tarjeta digitalizadora.

Fuente: autores.

Se carga el código en el PIC mediante el uso de la herramienta Pickit 3. Se verifica el funcionamiento de la tarjeta realizando los respectivos ajustes para adecuarla a la implementación en un circuito impreso (PCB). Haciendo uso del esquema que se muestra en la Fig. 3 se diseña el PCB en la herramienta Ares de Proteus. Se realizaron diferentes pruebas para corroborar el funcionamiento del impreso, una de ellas consistió en obtener el comportamiento en la lectura de la temperatura, a medida que ésta se incrementa o disminuye, donde la temperatura del LM35 refleja la temperatura ambiente y el valor sensado por la termocupla es 
el que varía. Posteriormente, se comparó los valores de la termocupla y el sensor LM35 cuando se someten a la misma temperatura para observar la respuesta de los dos sensores. Además, algunas pruebas se realizaron en condiciones controladas donde se calentó y enfrió agua en un recipiente sumergiendo tanto la termocupla didáctica que se usa con el prototipo, como una termocupla que se emplea con un datalogger digital patronado como referencia, se recibieron algunos datos por intervalos de tiempo de 30 minutos con ratas de muestreo variables desde 1 a 240 segundos.

En segundo lugar, las pruebas se efectuaron en campo. Para ello se instaló el prototipo paralelamente a una tarjeta industrial, marca Gamatec, que permite la adquisición de los datos de temperatura de la estación Termocupla Mallama del área de influencia del volcán Azufral en Nariño. La calibración y configuración de la tarjeta se hace con base en el datalogger digital utilizado por expertos en el área. Una vez adquiridos los datos en sede, se grafican los resultados, se computan diferencias absolutas entre las mediciones, valores máximos y mínimos, y se calculan promedios. Finalmente se establece el error promedio en el que está la medición de temperatura de la termocupla tanto en sede como en campo, con el fin de definir el comportamiento del prototipo.

\section{Resultados}

La depuración del código resultó eficiente gracias a la facilidad de compilación que ofrece el entorno de Mplab. En cuanto a los resultados de la simulación, se corroboró la concordancia entre los datos recibidos en la consola virtual y las señales medidas, además la tarjeta reaccionó muy bien en la prueba de las funciones que debe cumplir el prototipo como son la selección de transmisión de datos, cambios de configuración, reset físico, entre otros.

La simulación permitió determinar el cumplimiento de los requerimientos establecidos para el prototipo y la optimización de los errores de diseño. Para la
Figura 4, se corrió una simulación del programa en la que el voltaje de alimentación, y los sensores LM35 y termocupla se fijaron en valores de $14.5 \mathrm{~V}, 950 \mathrm{mV}$ $\left(95^{\circ} \mathrm{C}\right)$ y $740 \mathrm{mV}\left(74^{\circ} \mathrm{C}\right)$, respectivamente, el funcionamiento fue óptimo.

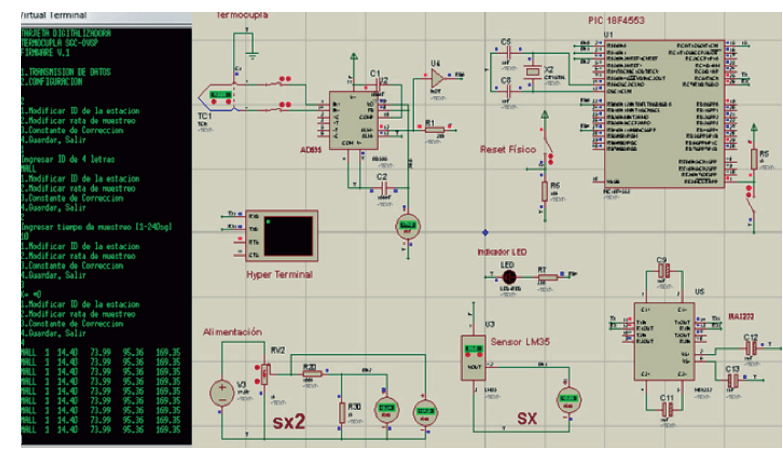

Fig. 4. Simulación del diseño de la tarjeta digitalizadora en el software Proteus. A la izquierda en consola, se aprecia el menú inicial y el de configuración con los parámetros con los que se ejecutó la simulación y algunos datos adquiridos, y a la derecha el entorno de simulación.

Fuente: autores.

Posteriormente, con el montaje en protoboard también se verificó el funcionamiento del prototipo, por lo que se determinó con certeza que en el montaje final el desempeño era el adecuado. En la Figura 5 se observa la reacción de la medida frente al incremento y descenso de la temperatura, que permite apreciar que el proceso de digitalización está funcionando correctamente. Por su parte, el diseño del PCB se muestra en la Figura 6 y su implementación en la Figura 7.
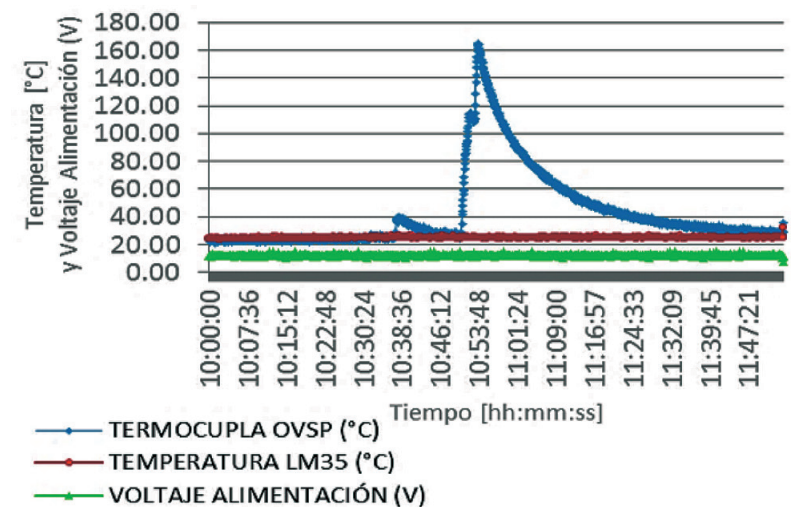

Fig. 5. Aumento y disminución de la temperatura de la termocupla, digitalizada con el prototipo. Prueba en laboratorio de la implementación en protoboard. Fuente: autores. 


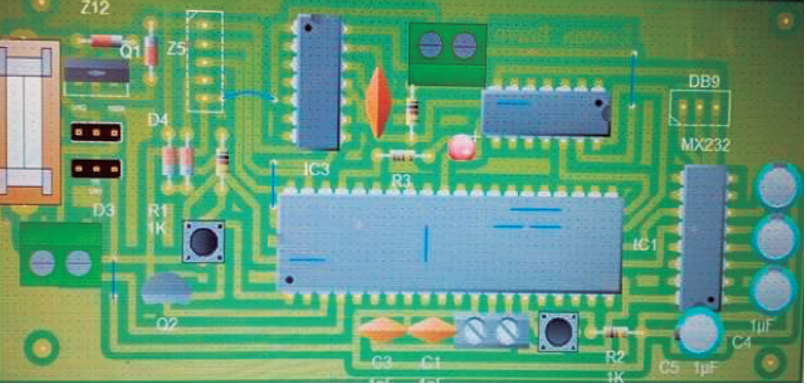

Fig. 6. Diseño del PCB del prototipo en el entorno Ares de Proteus.

Fuente: autores.

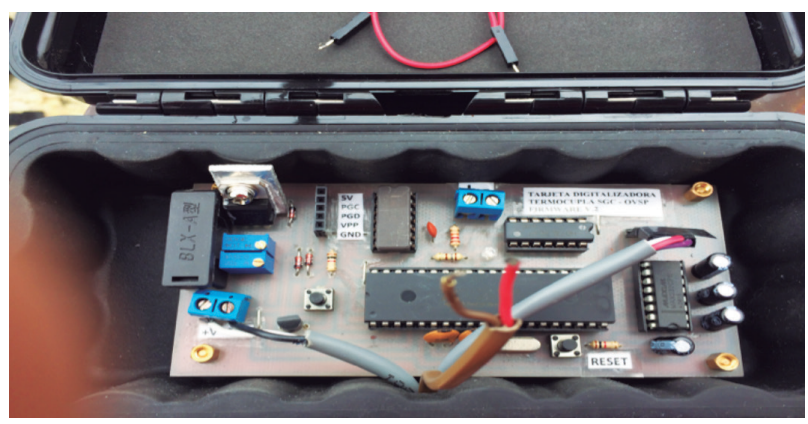

Fig. 7. Implementación del prototipo en el circuito impreso con su respectiva caja de protección IP67 para instalación en campo.

Fuente: autores.

\subsection{Pruebas en sede}

En cuanto al chequeo de conexiones de la implementación en PCB, se encontró que el montaje estaba en condiciones óptimas de funcionamiento. En la Figura 8 se observa el comportamiento de los dos sensores frente al incremento y descenso de la temperatura.

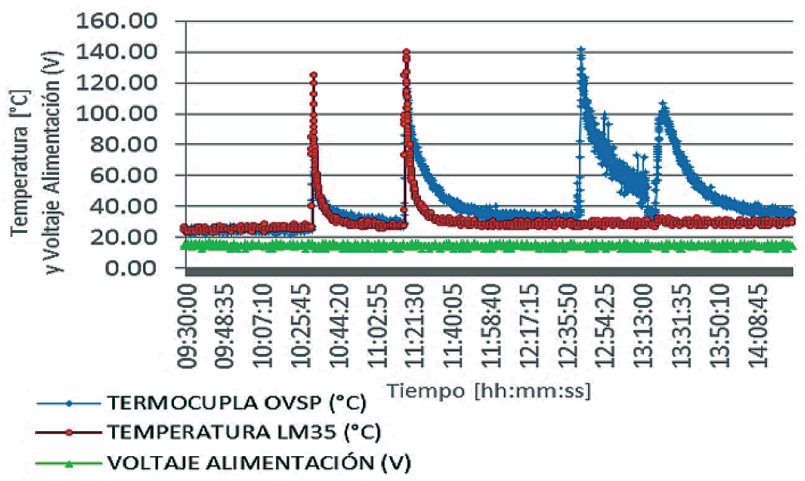

Fig. 8. Relación entre la medida de temperatura de la termocupla y del sensor LM35 digitalizadas con el prototipo. Prueba en laboratorio.
Se observó que la reacción de la termocupla frente al LM35 es relativamente igual cuando la temperatura aumenta, pero en la disminución de esta, la termocupla responde más lento que el LM35, por lo que es importante considerar que los cambios en la temperatura aportan al error en las mediciones debido al tiempo de reacción del instrumento de medida. Además en la Figura 9 se aprecia el comportamiento en la medición de temperatura en el rango de $(20 \text { a } 35)^{\circ} \mathrm{C}$, mientras que en la Figura 10 en el rango de los $88^{\circ} \mathrm{C}$ a los $94^{\circ} \mathrm{C}$. Se determinó que la tarjeta se comporta mejor en las bajas temperaturas, probablemente porque en estos casos, normalmente la salida del acondicionamiento de señal está sujeta a calibración, ganancia y sensibilidad a la temperatura asociada, en este caso a un incremento de la misma. Además, el contacto térmico con el medio a medir presenta una resistencia térmica que enmascara la medida, y que puede variar con el tiempo.

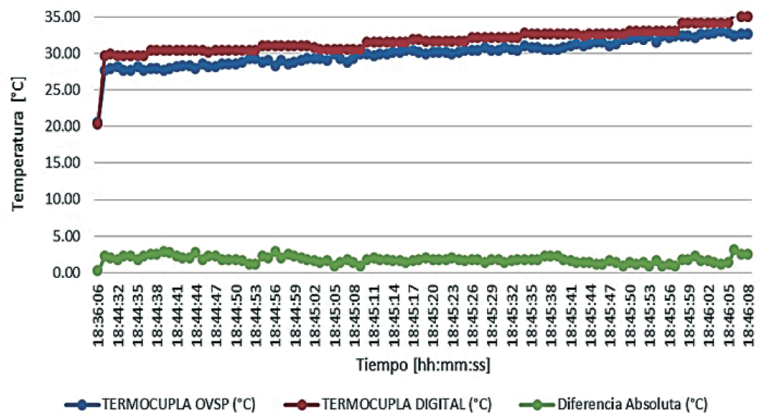

Fig. 9. Comparación entre la medida de temperatura con el prototipo de la termocupla didáctica (Termocupla OVSP) y con el datalogger digital de la termocupla de referencia (Termocupla Digital), en el rango de $20^{\circ} \mathrm{C}$ a $35^{\circ} \mathrm{C}$.

Fuente: autores.

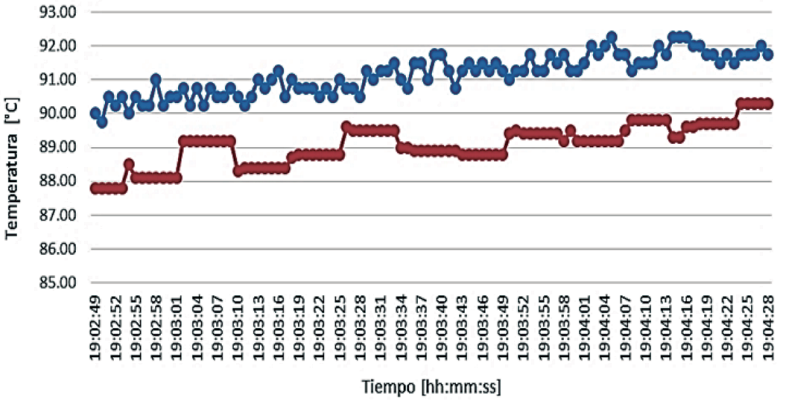

Fig. 10. Comparación entre la medida de temperatura con el prototipo de la termocupla didáctica (Termocupla OVSP) y con el datalogger digital de la termocupla de referencia (Termocupla Digital), en el rango de $88^{\circ} \mathrm{C}$ a $94^{\circ} \mathrm{C}$. 
Como última prueba en sede, el prototipo se energizó y se dejó funcionando por un periodo de 2 días, sin inconveniente alguno en la prueba y obteniendo mediciones acertadas con un error absoluto de $1^{\circ} \mathrm{C}$.

\subsection{Pruebas en campo}

La tarjeta se instaló con la configuración: ID: MALL, Rata de muestreo: 60 segundos (se recibe un dato cada minuto), y constante de corrección: $\mathrm{K}=0$. Desde el SGC-OVSP se monitoreó su comportamiento, se realizaron varias gráficas de los datos adquiridos, en las que se encontró un funcionamiento relativamente bueno, sin embargo hubo ocasiones en las que se recibieron datos erróneos con valores de temperatura para la termocupla de entre $100^{\circ} \mathrm{C}$ y $600^{\circ} \mathrm{C}$ que pueden deberse al acondicionamiento de señal, al verse afectado por las condiciones del entorno en el que se instala la tarjeta, corrosión, temperatura, acidez, entre otros. En la Figura 11 se aprecia una gráfica de los datos adquiridos en sede durante las 24 horas de un día, un total de 1.440 datos.

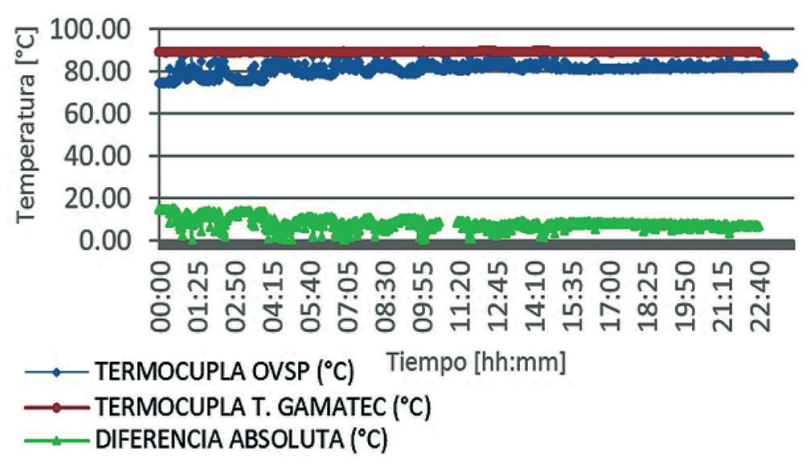

Fig. 11. Paralelo entre la medida de temperatura de la termocupla digitalizada con el prototipo y la termocupla cuyos datos se adquieren con la tarjeta Gamatec. Prueba en campo para un día completo.

Fuente: autores.

Aunque las dos tarjetas se encuentran instaladas en campos fumarólicos diferentes, pero relativamente cercanos (10m de separación física), se puede hacer un paralelo entre los datos de temperatura adquiridos para observar el comportamiento de los dos procesos de digitalización. La tarjeta Gamatec aporta un valor constante, mientras que el prototipo diseñado presenta saltos bruscos en las mediciones.
En general, los datos adquiridos en campo durante ese periodo de tiempo presentaron estabilidad en algunas ocasiones y fluctuaciones en otras, el error promedio obtenido fue de $+/-2^{\circ} \mathrm{C}$. La tarjeta permanece instalada en campo y se continúa monitoreando su funcionamiento con el propósito de detectar errores y proponer una futura optimización del diseño, con el fin de conseguir la tarjeta digitalizadora del OVSP que pueda ser instalada en otras estaciones de vigilancia volcánica del instituto.

\section{Conclusiones y trabajo futuro}

El error obtenido en las mediciones se debe principalmente a la juntura térmica que debe tener el sensor con el medio, la cual presenta una resistencia que enmascara la medida y que además puede variar en el tiempo.

Es necesario optimizar la etapa de hardware del diseńo propuesto, adicionando protecciones para evitar interferencia y ruido eléctrico inducido por la etapa de potencia, y cambios bruscos en las mediciones debidos a variaciones de tensión externas que afectan a los componentes más sensibles del circuito.

Se considera que el prototipo realizado es susceptible de mejorar en cuanto a la calidad y robustez de los compontes electrónicos empleados, puesto que las condiciones adversas en las que se instala (corrosión, acidez, humedad, temperatura etc.), tienen una marcada influencia en el registro de los datos.

\section{REFERENCIAS}

Adeyeri, M. K., Kareem, B., Adeyemi, A. A. \& Adewale, S. O. (2012). Temperature based embedded programming algorithm for conventional machines condition monitoring. Computation tools (2012), IARIA. pp. 51-57. Recuperado en: https:// www.thinkmind.org/download.php?articleid=computation tools_2012_2_50_8030059

Carrion, C. J. (2015). Implementación de un termómetro digital para medir la temperatura de una piscina camaronera utilizando un microcontrolador tipo PIC 16f84a. Machala: Unidad Académica de Ingeniería Civil, Carrera de Ingeniería de Sistemas. 
Andrea Lorena Bravo Suarez, Jorge Edison Mora Arroyo

Diseño e implementación de un prototipo de tarjeta digitalizadora para monitoreo

termométrico en fuentes termales y campos fumarólicos en áreas de influencia volcánica

Firtec (s.f.). La EUSART en el C18 (Enhanced Universal Synchronous Receiver Transmitter. Librerías del compilador C18, usart functions, Recuperado en: http://firtec.com.ar/apuntes/uart. zip?hc_location=ufi

López, L. A., Molina, S. O. \& Vídes, R. A. (2007). Desarrollo de un sistema de monitoreo volcánico. San Salvador: Universidad de El Salvador, Facultad de Ingeniería y Arquitectura, Escuela de Ingeniería Eléctrica.

Meléndez, R. (2008). Sistema autónomo de medición de variables de temperatura y humedad relativa en un secador solar para madera con capacidad de transmitir los datos vía USB a un dispositivo flash. Cartago: Instituto Tecnológico de Costa Rica, Escuela de Ingeniería Electrónica.
Microchip (2007). PIC18F2458/2553/4458/4553 - Data Sheet. 28/40/44-Pin High-Performance, Enhanced Flash, USB Microcontrollers with 12-Bit A/D and nanoWatt Technology. Microchip Technology Inc. Recuperado en: http://www.mouser.com/ ds/2/268/39887b-62277.pdf

Schmid, W. A. \& Lazos, R. J. (2004). Guia para estimar la incertidumbre de la medición. El Marqués, Qro.: Centro Nacional de Metrología CENAM.

Quijada, O. M. \& Argueta, A. (2011). Diseño y construcción de un instrumento electrónico para cuantificar magnitudes físicas (Data Logger). Primera etapa: medir la temperatura. San Salvador: Universidad de El Salvador, Facultad de Ingeniería y Arquitectura, Escuela de Ingeniería Eléctrica. 


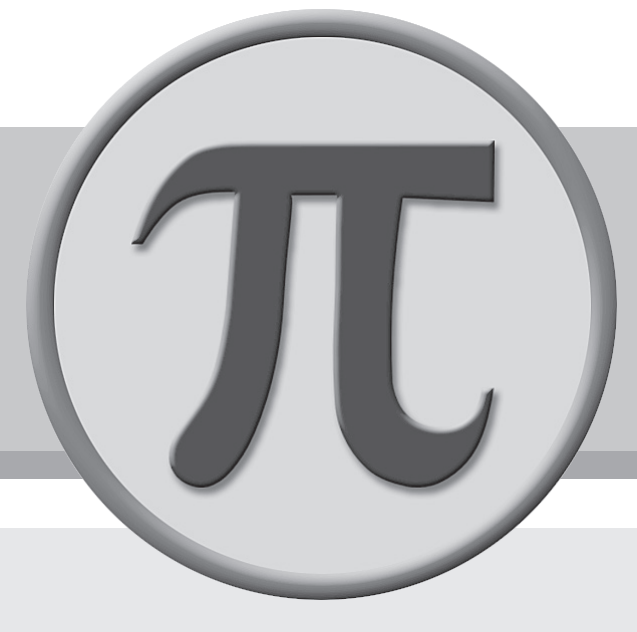


